\title{
Autoinflammatory Syndrome
}

National Cancer Institute

\section{Source}

National Cancer Institute. Autoinflammatory Syndrome. NCI Thesaurus. Code C119050.

A group of disorders of the innate immune system characterized by attacks of seemingly unprovoked inflammation without significant levels of either autoantibodies or autoreactive T cells more characteristic of autoimmune disease. 